\title{
28 Research Square \\ PET/CT Radiomics in Breast Cancer: Promising Tool for the Prediction of the Ki67 Expression
}

\section{Cong Shen}

The First Affiliated Hospital of Xi'an Jiaotong University

\section{Dong Han}

Affiliated Hospital of Shaanxi Unviersity of Chinese Medicine of

\section{Yunxuan Li}

The First Affiliated Hospital of Xi'an Jiaotong University

\section{Anqi Zheng}

The First Affiliated Hospital of Xi'an Jiaotong University

\section{Qi Nie}

Philips Healthcare

\section{Chengyu Ding}

Philips Healthcare

Xiaoyi Duan ( $\nabla$ duanxyi@yeah.net )

The First Affiliated Hospital of Xi'an Jiaotong University

\section{Original research}

Keywords: PET/CT, radiomics, breast cancer, Ki67

Posted Date: July 22nd, 2021

DOI: https://doi.org/10.21203/rs.3.rs-707398/v1

License: (c) (i) This work is licensed under a Creative Commons Attribution 4.0 International License.

Read Full License 


\section{Abstract}

Objective: This study aims to examine the values of radiomics parameters derived from ${ }^{18}$-fluorinefluorodeoxyglucose $\left.{ }^{(18} \mathrm{F}-\mathrm{FDG}\right) \mathrm{PET} /$ computed tomography (CT) imaging in the prediction of ki-67 expression in breast cancer patients.

Patients and methods: A total of 115 patients diagnosed with breast cancer and examined by ${ }^{18} \mathrm{~F}-\mathrm{FDG}$ PET/CT were included in this study. The Ki-67 proliferation index was determined from the pathological specimen as positive or negative. Radiomics features were extracted by pyRadiomics and reduced by Independent $t$-test and least absolute shrinkage selection operator. The radiomics risk score (RRS) was calculated with all the selected features. RRS incorporated with clinical-pathological features were used to construct a binary logistic regression and nomogram classifier. Receiver operating characteristic curve (ROC) analysis was used to predict the accuracy. Decision curve analysis (DCA) was performed to assess clinical utility.

Results: Totally 944 features were reduced to 14 predictors. RRS were significantly differed between the ki67+ and ki67-groups $(0.440 \pm 0.473$ and $1.039 \pm 0.430 ; t=-6.663, p<0.001)$. In the binary logistic regression, $\mathrm{N}$ stage (OR [95\% Cl], 5.752 [2.032, 16.286], $p<0.001)$ and RRS (OR [95\%Cl], 20.540 [5.521, 76.423], $p<0.001)$ were independent factors in predicting Ki67 expression. In ROC analysis, AUC was $0.866(0.790,0.922),(p<0.001)$, with sensitivity, specificity, Youden index and cutoff value of $82.50 \%$, $80.00 \%, 0.6250$ and 0.6672 , respectively. DCA indicated that use of the clinical-radiomic nomogram had more benefit than utilizing either clinical or radiomic features alone.

Conclusion: The radiomics-derived evaluation score combined with $\mathrm{N}$ stage could effectively predict Ki67 expression in breast cancer, enabling proper patient selection for treatment.

\section{Background}

Breast cancer has become the highest incident tumor in women, with high morbidity and mortality worldwide ${ }^{1}$. Breast cancer is a heterogeneous disease that comprises different molecular subtypes characterized by diverse histological characteristics, therapeutic strategies and prognostic implications ${ }^{2}$. With the advent of new treatments, it becomes important to individualize therapy according to the biomarker status of the tumor.

Ki67 is the most commonly used biomarker to evaluate the proliferative index of breast cancer. Several studies found that high Ki67 is associated with elevated rate of relapse and worse survival in breast cancer $^{3-6}$. Studies have demonstrated the clinical validity of Ki-67 as a predictive marker in the neoadjuvant setting ${ }^{7}$. Given that Ki67 is principally used for estimating prognosis and decision guiding regarding adjuvant treatment, Ki67 stratification and prediction are important. 
${ }^{18} \mathrm{~F}$-fluorodeoxyglucose $\left({ }^{18} \mathrm{~F}-\mathrm{FDG}\right)$ positron emission tomography/computed tomography (PET/CT) is widely and routinely used in breast cancer staging ${ }^{8}$. Standard semi-quantitative imaging parameters obtained from ${ }^{18} \mathrm{~F}$-FDG PET/CT have been shown to correlate with tumor aggressiveness and patient outcome in breast cancer ${ }^{9-12}$. Radiomics, an approach that can quantify lesion heterogeneity and texture, holds promise in addressing clinical challenges in monitoring of disease progression ${ }^{13}$. Recent reports indicated that features obtained from ${ }^{18} \mathrm{~F}$-FDG PET/CT are associated with the tumor's histological characteristics and molecular subtypes ${ }^{14-16}$, but limited evidence relating to their roles as predictive parameters is available.

The main objective of this study was to evaluate the roles of radiomics-derived imaging features obtained from baseline ${ }^{18} \mathrm{~F}$-FDG PET/CT in combination with clinical parameters in the prediction of Ki67 expression in breast cancer patients.

\section{Materials And Methods}

\subsection{Study population}

This retrospective study was approved by the Ethics Committee of the First Affiliated Hospital of Xi'an Jiaotong University, and informed consent was waived. A total of 129 female patients who underwent ${ }^{18}$ F-FDG PET/CT examinations for breast cancer in our hospital from November 2016 to June 2020 were retrospectively analyzed. Inclusion criteria were: $\otimes$ breast cancer confirmed by preoperative puncture or postoperative pathologically within 2 weeks; $邓$ with Ki67 expression records; $₫$ underwent 18F-FDG PET/CT

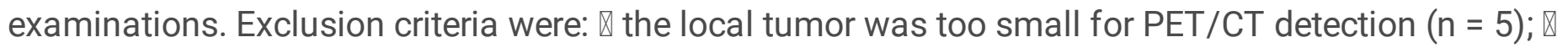
diffuse or multifocal lesion in unilateral or bilateral breasts $(n=5)$; $\otimes$ neo-adjuvant chemotherapy or antitumor treatment performed before imaging $(n=4)$.

The flowchart is shown in Figure 1. A total of 115 patients were enrolled in this study. Their basic characteristics are listed in Table 1.

\subsection{Immunohistochemistry}

Formalin-fixed paraffin-embedded tissue samples from breast cancer cases were used for Ki67 assessment by an experienced pathologist, who was blinded to PET/CT results. Ki-67 levels were divided into the $0.5 \%, 10 \%, 15 \%, 20 \%, 30 \%, 40 \%, 50 \%, 60 \%, 70 \%, 80 \%, 90 \%$ and $100 \%$ groups according to the degree of expression, and expression index $\geq 15 \%$ was considered positive, and vice versa.

\subsection{PET/CT data acquisition}

All examinations were performed on a 64-detector scanner (Gemini TF PET/CT, Philips, Netherlands). ${ }^{18} \mathrm{~F}$ FDG was synthesized with a small cyclotron (GE MINItrace) and an FDG synthesis module. The radiochemical purity was $>99 \%$. Both endotoxin and bacteriological tests were negative, which met the radiopharmaceutical requirements. 
The patients fasted for more than 6 hours before the intravenous injection of ${ }^{18} \mathrm{~F}$-FDG. Fasting blood glucose should be lower than $12.0 \mathrm{mmol} / \mathrm{L}$ at the administration of ${ }^{18} \mathrm{~F}-\mathrm{FDG}(3.7 \mathrm{MBq} / \mathrm{kg})$. After resting for 60 minutes, the patients were asked to perform whole body PET/CT. The scan scope was from the top of the skull or the level of the first thoracic vertebrae to the upper femur. CT scans (tube voltage, $120 \mathrm{kV}$ and automatic milliampere second; matrix, $512 \times 512$; layer thickness, $5 \mathrm{~mm}$ ) were performed for the corresponding layers. PET collects $7-10$ beds with $1.5 \mathrm{~min} / \mathrm{bed}$. Attenuation correction was performed on each PET image by CT, and the iterative method was used for reconstruction. MIP, PET, CT and fusion images were displayed on the EBW workstation.

\subsection{Image analysis}

The volume of interest (VOI) was automatically delineated with SUVmax $40 \%$ as the threshold on the Philips workstation. The VOIs were reviewed by an expert (Cong Shen), and inaccurate VOls were manually corrected. Maximum standardized uptake (SUVmax), mean standard uptake (SUVmean), metabolic tumor volume (MTV) and total lesion glycolysis (TLG) were calculated automatically.

Two experts (Xiaoyi Duan, reader 1; Cong Shen, reader 2) with more than 10 years of PET/CT interpretation experience determined $\mathrm{N}$ and $\mathrm{M}$ stage cases in a double-blind manner. Any disagreement between the two radiologists was resolved by another, more experienced radiologist.

\subsection{Feature extraction}

The VOIs were then saved as .nii files. Radiomics feature extraction was implemented using the Philips Radiomics Tool (Philips Healthcare, China); the core feature calculation was based on pyRadiomics ${ }^{17}$. A total of 944 three-dimensional (3D) radiomic parameters, including direct, wavelet transform, logarithm transform, and gradient filtered features, were extracted. Details of the feature extraction was showed in the Supplementary.

\subsection{Statistical analysis}

Data were analyzed with SPSS ${ }^{\circledR}$ v. 25.0. (IBM Corp., New York, NY) and R v. 3.5.2 (Vienna, Austria. URL https://www. R-project. org/). $P<0.05$ indicated statistically significant differences.

Continuous variables with normal distribution and homogeneity of variance were expressed as mean \pm standard deviation and tested by independent samples Student's $t$-test; otherwise, the Mann-Whitney $U$ test was used. Categorical variables were compared by the $\chi 2$ test or the Fisher's exact test.

In order to prevent model over-fitting, the two-sample $t$ test and least absolute shrinkage and selection operator (LASSO) regression were used to select the most discriminative features by the glmnet package in R. The LASSO regression model was selected with the optimal $\lambda$ value by the cross-validation method. A Radiomics Risk Score (RRS) ${ }^{13}$ is an indicator of all discriminative features and is determined by the LASSO regression. 
With Ki67 grading as the reference standard, binary logistic regression was used to construct prediction models. The predictive accuracies of independent factors selected by binary logistic regression and the nomogram classifier were assessed by receiver operating characteristic curve (ROC) analysis, determining the area under the curve (AUC), C-statistics, sensitivity and specificity. The HosmerLemeshow goodness-of-fit test was used to evaluate the calibration of the models. Decision curve analysis (DCA) was performed to assess the clinical utility of the prediction model by quantifying the patients' net benefits under different probability thresholds.

\section{Results}

\subsection{Clinical characteristics}

The clinical characteristics of the patients are summarized in Table 1. There were no significant differences between the 2 cohorts in terms of age, lesion location, status of menstruation and $M$ stage. In addition, a few clinical characteristics were significantly different between the two groups, including $\mathrm{N}$ stage, tumor size, SUVmax, SUVmean, SD and MTV (Table 1).

\subsection{Feature extraction and RRS calculation}

Totally 944 features were reduced to 14 potential predictors by applying Student's $t$-test and regularized regression to the primary cohort with the LASSO penalty via minimum criteria (Fig. 2).

The RRS were calculated by the selected features and LASSO regression, as indicated by the following equation. The RRS were significantly different between the two groups $(0.440 \pm 0.473$ and $1.039 \pm 0.430$ in the ki67+ and ki67-groups, respectively; $t=-6.663, p<0.001)$.

RRS = -ZwaveletLHL_gldm_SmallDependenceHighGrayLevelEmphasis * 0.247327621 -

ZwaveletLHH_firstorder_Median * 0.225384311 -ZwaveletLHH_glrlm_ShortRunHighGrayLevelEmphasis * 0.076456158 -ZwaveletHHL_glszm_GrayLevelNonUniformityNormalized * 0.064495061 -

ZwaveletHHH_glszm_GrayLevelNonUniformityNormalized * 0.040682893-

ZwaveletLHH_gldm_HighGrayLevelEmphasis * 0.010784806-ZwaveletHHL_ngtdm_Contrast *

0.005186627-ZwaveletHLH_firstorder_Range *

$0.031249748+Z$ waveletLHH_glszm_SmallAreaLowGrayLevelEmphasis *

$0.039624596+Z o r i g i n a l \_g l d m \_L a r g e D e p e n d e n c e L o w G r a y L e v e l E m p h a s i s$ *

$0.047298516+Z$ logsigma60mm3D_gldm_LargeDependenceLowGrayLevelEmphasis *

$0.089515333+Z$ logsigma60mm3D_firstorder_Skewness *

$0.093296711+Z$ waveletLHL_glrlm_GrayLevelNonUniformityNormalized *

$0.11338656+Z$ original_firstorder_Maximum * $0.123449315+0.856591991$

\subsection{Binary logistic regression}

Of all clinical parameters, $\mathrm{N}$ stage (OR [95\% Cl], 5.752 [2.032, 16.286], $p<0.001)$ and RRS (OR [95\% Cl], $20.540[5.521,76.423], p<0.001)$ were independent factors predicting Ki67 expression (Table 2). A clinical- 
radiomic nomogram that incorporated these independent predictors was developed (Figure 3).

\subsection{ROC analysis and clinical application}

In ROC analysis, the AUC was $0.866(0.790,0.922)(p<0.001)$, with sensitivity, specificity, Youden index and cutoff value of $82.50 \%, 80.00 \%, 0.6250$ and 0.66718 , respectively (Figure 4). DCA indicated that use of the clinical-radiomic nomogram had more benefit than utilizing the clinical or radiomic features alone(Figure 5).

\section{Discussion}

The evaluation of prognostic and predictive factors is very important for the identification of patients at high risk of recurrence, and for choosing the most appropriate treatment for individual patients. Radiomics is a newly introduced image-analysis method involving high-throughput features extracted from radiographic images, which is promising in the prediction of tumor heterogeneity because of its noninvasive and repeatable properties.

This study showed the application of radiomics in Ki67 prediction in breast cancer. Combining the $\mathrm{N}$ stage and radiomics risk score, the AUC was 0.866 in the prediction of Ki67 expression in breast cancer, with sensitivity, specificity, Youden index and cutoff value of $82.50 \%, 80.00 \%, 0.6250$ and 0.66718 , respectively $(0.790,0.922)(p<0.001)$. A novel nomogram incorporating radiomic and clinical features was developed, providing an easy-to-use method in the prediction of ki-67 expression. DCA displayed a greater net benefit of the combination of clinical and radiomics features compared with either of them alone. This strategy may have clinical implications for individualized follow-up and guiding therapeutic strategies.

Recent studies showed that multiple semi-quantitative and volumetric parameters on ${ }^{18} \mathrm{~F}$-FDG PET/CT images are correlated with $\mathrm{Ki}-67$ expression ${ }^{18,19}$. Besides those volumetric parameters, textural findings may improve in terms of predicting treatment response and determining the likelihood of metastasis/recurrence. A recent study showed that the ki-67 status does not differ in any of the textual analyse ${ }^{20}$. However, in this study, multiple textural features showed differences between the ki67 + and ki67+- groups. The most possible reasons were as follows. (1) A different method was used for feature extraction. Most of the parameters were wavelet features in this study. (2) More cases were included in this study, which may give firmer conclusions.

This study had several limitations. Firstly, all primary breast cancer cases without treatment before PET/CT examination were from only one center. Thus, the sample size was small, which precluded the establishment of training and validation cohorts. More breast cancer cases should be included, preferentially from different centers, to obtain stable algorithms. Secondly, there was low unbalance of cases with positive Ki-67 and negative ki67 expression due to the retrospective nature of the study. 


\section{Conclusion}

The radiomics-derived evaluation score combined with the $\mathrm{N}$ stage could effectively predict the Ki67 expression in breast cancer based on PET/CT images, enabling proper patient selection for treatment. However, further calibration and validation in high-quality prospective studies are required.

\section{Abbreviations}

${ }^{18}$ F-FDG, 18-fluorine-fluorodeoxyglucose

DCA, Decision curve analysis

LASSO, least absolute shrinkage and selection operator

MTV, metabolic tumor volume

PET/CT, positron emission tomography/computed tomography

ROC, Receiver operating characteristic

RRS, radiomics risk score

SUVmax, maximum standardized uptake

SUVmean, mean standard uptake

TLG, total lesion glycolysis

VOI, volume of interest

\section{Declarations}

\section{Declaration of Interest Statement:}

1.Ethics approval and consent to participate

This study was approved by the Ethics Committee of the First Affiliated Hospital of Xi'an Jiaotong University, and informed consent was waived.

2.Consent for publication

Written informed consent for publication was obtained from all participants.

3.Availability of data and material 
We confirm that all the materials and data with regard to the analysis in the manuscript are available for request.

\section{Competing interests}

The authors report no conflicts of interest.

\section{Funding}

This study was funded by National Natural Science Foundation of China (62073260).

\section{Authors' contributions}

Cong Shen, Qi Nie and Xiaoyi Duan contributed to the conception of the study; Cong Shen, Dong Han, Yunxuan Li, Anqi Zheng, Qi Nie and Chengyu Ding performed the experiment; Cong Shen, Dong Han and Chengyu Ding contributed significantly to analysis and manuscript preparation; Cong Shen, Qi Nie and Xiaoyi Duan helped perform the analysis with constructive discussions.

\section{Acknowledgements}

Not appliable.

\section{References}

1. Siegel RL, Miller KD, Jemal A. Cancer statistics, 2020. CA: a cancer journal for clinicians. 2020;70(1):730 .

2. Perou $\mathrm{CM}$, Sorlie T, Eisen MB, et al. Molecular portraits of human breast tumours. Nature. 2000;406(6797):747-752.

3. Baker E, Whiteoak N, Hall L, France J, Wilson D, Bhaskar P. Mammaglobin-A, VEGFR3, and Ki67 in Human Breast Cancer Pathology and Five Year Survival. Breast cancer : basic and clinical research. 2019;13:1178223419858957.

4. Gallardo A, Garcia-Valdecasas B, Murata P, et al. Inverse relationship between Ki67 and survival in early luminal breast cancer: confirmation in a multivariate analysis. Breast cancer research and treatment. 2018;167(1):31-37.

5. Kilickap S, Kaya Y, Yucel B, Tuncer E, Babacan NA, Elagoz S. Higher Ki67 expression is associates with unfavorable prognostic factors and shorter survival in breast cancer. Asian Pacific journal of cancer prevention : APJCP. 2014;15(3):1381-1385.

6. Ishihara M, Mukai $\mathrm{H}$, Nagai $\mathrm{S}$, et al. Retrospective analysis of risk factors for central nervous system metastases in operable breast cancer: effects of biologic subtype and Ki67 overexpression on survival. Oncology. 2013;84(3):135-140. 
7. Acs B, Zambo V, Vizkeleti L, et al. Ki-67 as a controversial predictive and prognostic marker in breast cancer patients treated with neoadjuvant chemotherapy. Diagn Pathol. 2017;12:1-12.

8. Chakraborty D, Basu S, Ulaner GA, Alavi A, Kumar R. Diagnostic Role of Fluorodeoxyglucose PET in Breast Cancer: A History to Current Application. PET clinics. 2018;13(3):355-361.

9. Kitajima K, Miyoshi Y, Sekine T, et al. Harmonized pretreatment quantitative volume-based FDG-PET/CT parameters for prognosis of stage I-III breast cancer: Multicenter study. Oncotarget. 2021;12(2):95-105.

10. Kitajima K, Miyoshi Y, Sekine T, et al. Harmonized pretreatment quantitative volume-based (18)F-FDG PET/CT parameters for stage IV breast cancer prognosis. Multicenter study in Japan. Hellenic journal of nuclear medicine. 2020;23(3):272-289.

11. Gelezhe PB, Blokhin IA, Marapov DI, Morozov SP. Quantitative parameters of MRI and (18)F-FDG $\mathrm{PET} / \mathrm{CT}$ in the prediction of breast cancer prognosis and molecular type: an original study. American journal of nuclear medicine and molecular imaging. 2020;10(6):279-292.

12. Qu YH, Long N, Ran C, Sun J. The correlation of (18)F-FDG PET/CT metabolic parameters, clinicopathological factors, and prognosis in breast cancer. Clinical \& translational oncology : official publication of the Federation of Spanish Oncology Societies and of the National Cancer Institute of Mexico. 2021;23(3):620-627.

13. Tagliafico AS, Piana M, Schenone D, Lai R, Massone AM, Houssami N. Overview of radiomics in breast cancer diagnosis and prognostication. Breast. 2020;49:74-80.

14. Waks AG, Winer EP. Breast Cancer Treatment A Review. Jama-Journal Of the American Medical Association. 2019;321(3):288-300.

15. Antunovic L, Gallivanone F, Sollini M, et al. [(18)F]FDG PET/CT features for the molecular characterization of primary breast tumors. European journal of nuclear medicine and molecular imaging. 2017;44(12):1945-1954.

16. Khare S, Singh SS, Irrinki S, et al. (18)F-Fluorodeoxyglucose Positron Emission Tomography/Computed Tomography Features in Locally Advanced Breast Cancer and Their Correlation with Molecular Subtypes. Indian journal of nuclear medicine : IJNM : the official journal of the Society of Nuclear Medicine, India. 2018;33(4):290-294.

17. van Griethuysen JJM, Fedorov A, Parmar C, et al. Computational Radiomics System to Decode the Radiographic Phenotype. Cancer research. 2017;77(21):e104-e107.

18. Mohamadien NRA, Sayed MHM. Correlation between semiquantitative and volumetric 18F-FDG $\mathrm{PET} /$ computed tomography parameters and Ki-67 expression in breast cancer. Nucl Med Commun. 2021. 
19. Deng SM, Zhang W, Zhang B, Chen YY, Li JH, Wu YW. Correlation between the Uptake of F-18Fluorodeoxyglucose (F-18-FDG) and the Expression of Proliferation-Associated Antigen Ki-67 in Cancer Patients: A Meta-Analysis. Plos One. 2015;10(6).

20. Acar E, Turgut B, Yigit S, Kaya G. Comparison of the volumetric and radiomics findings of 18F-FDG $\mathrm{PET} / \mathrm{CT}$ images with immunohistochemical prognostic factors in local/locally advanced breast cancer. Nucl Med Commun. 2019;40(7):764-772.

\section{Tables}

Due to technical limitations, table 1-2 is only available as a download in the Supplemental Files section.

\section{Supplementary Materials}

Supplementary Materials are not available with this version.

\section{Figures}

\begin{tabular}{|c|c|c|}
\hline & $\begin{array}{l}\text { With PET/CT examination due to the } \\
\text { suspected or confirmed breast cancer } \\
\text { from November } 2016 \text { to June } 2020 \\
\text { (n }=129)\end{array}$ & \\
\hline & & $\begin{array}{l}\text { (1) the lesion is too small }(n=5) \text {; } \\
\text { (2) diffuse or multifocal lesions }(n=5) \text {; } \\
\text { (3) with neo-adjuvant chemotherapy ol } \\
\text { anti-tumor treatment before imaging } \\
(n=4) \text {. }\end{array}$ \\
\hline \multicolumn{2}{|c|}{ Breast cancer for analysis $(n=115)$} & \\
\hline $\mathrm{Ki} 67+(\mathrm{n}=80)$ & Ki67- & $=35)$ \\
\hline
\end{tabular}

Figure 1

Study flowchart 

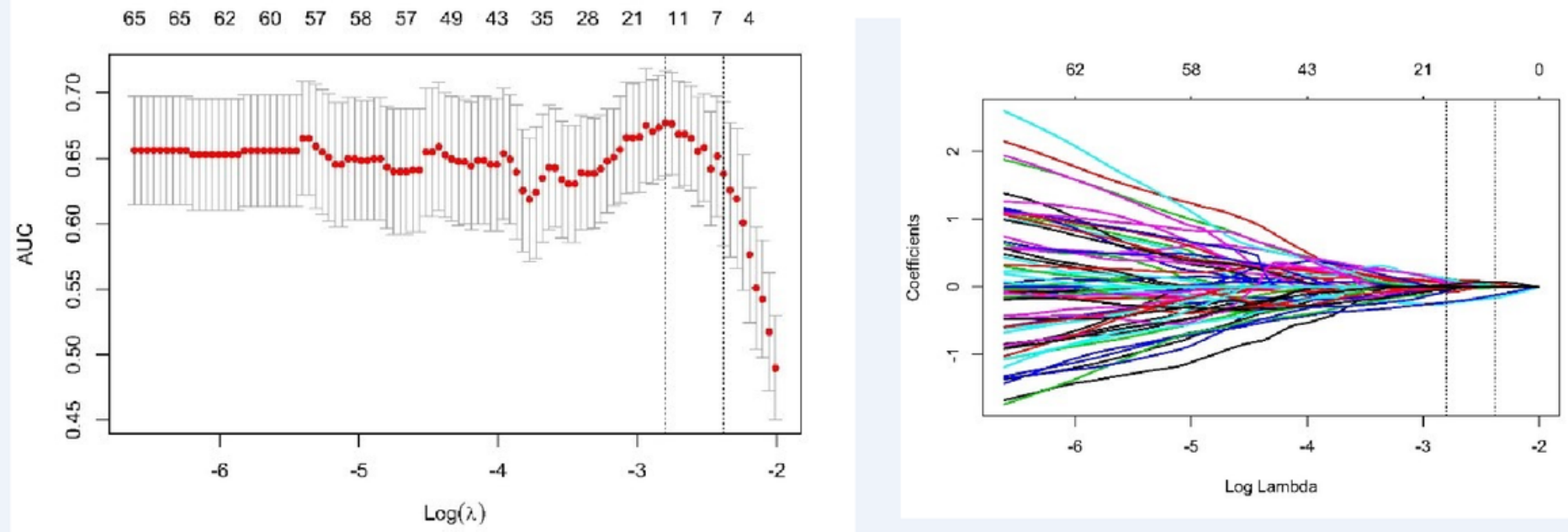

Figure 2

Feature selection with the least absolute shrinkage and selection operator A dotted vertical line was drawn at the optimal $\lambda$ by using the minimum mean-squared error. A value of 0.06090181 was chosen as $\lambda$, and 14 features were selected.

Points

N_stage

Radiomics_score

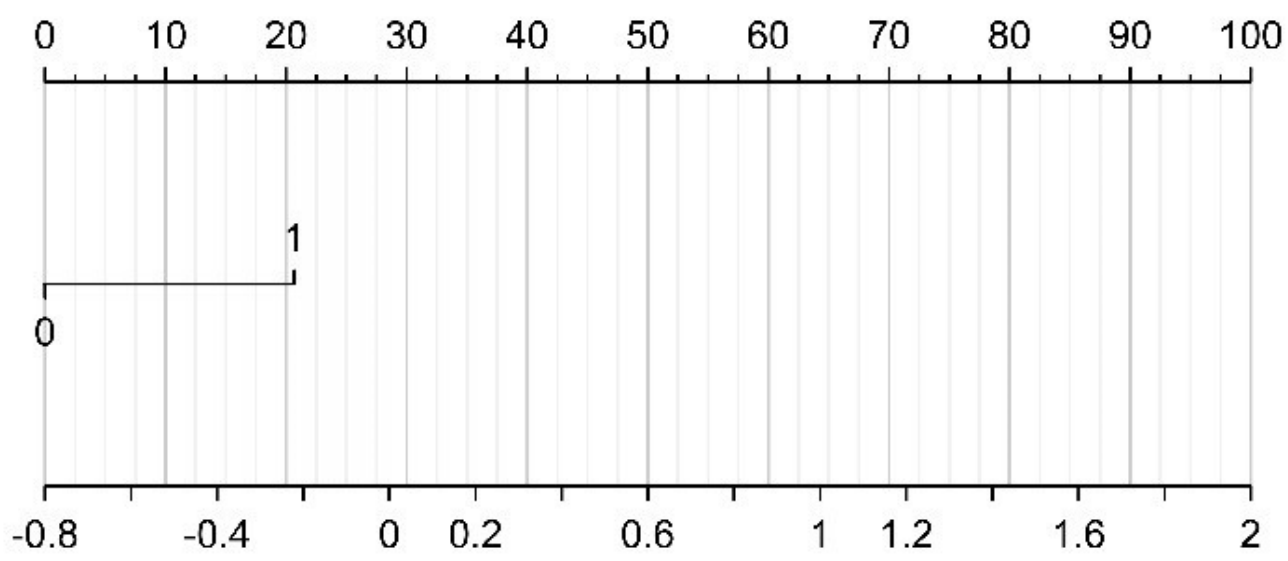

Total Points

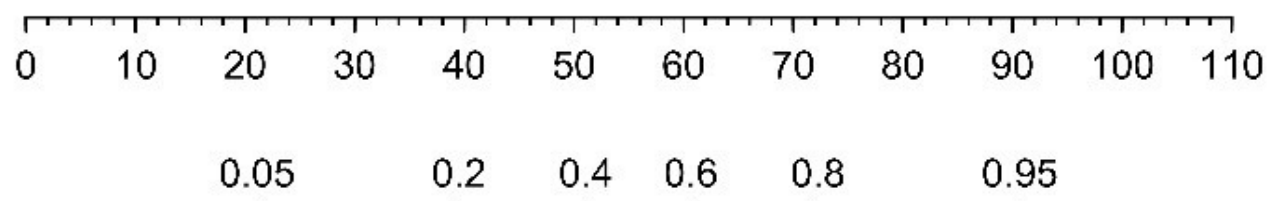

Ki-67 (+)

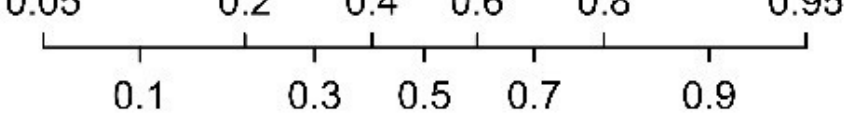


Figure 3

The developed radiomics nomogram
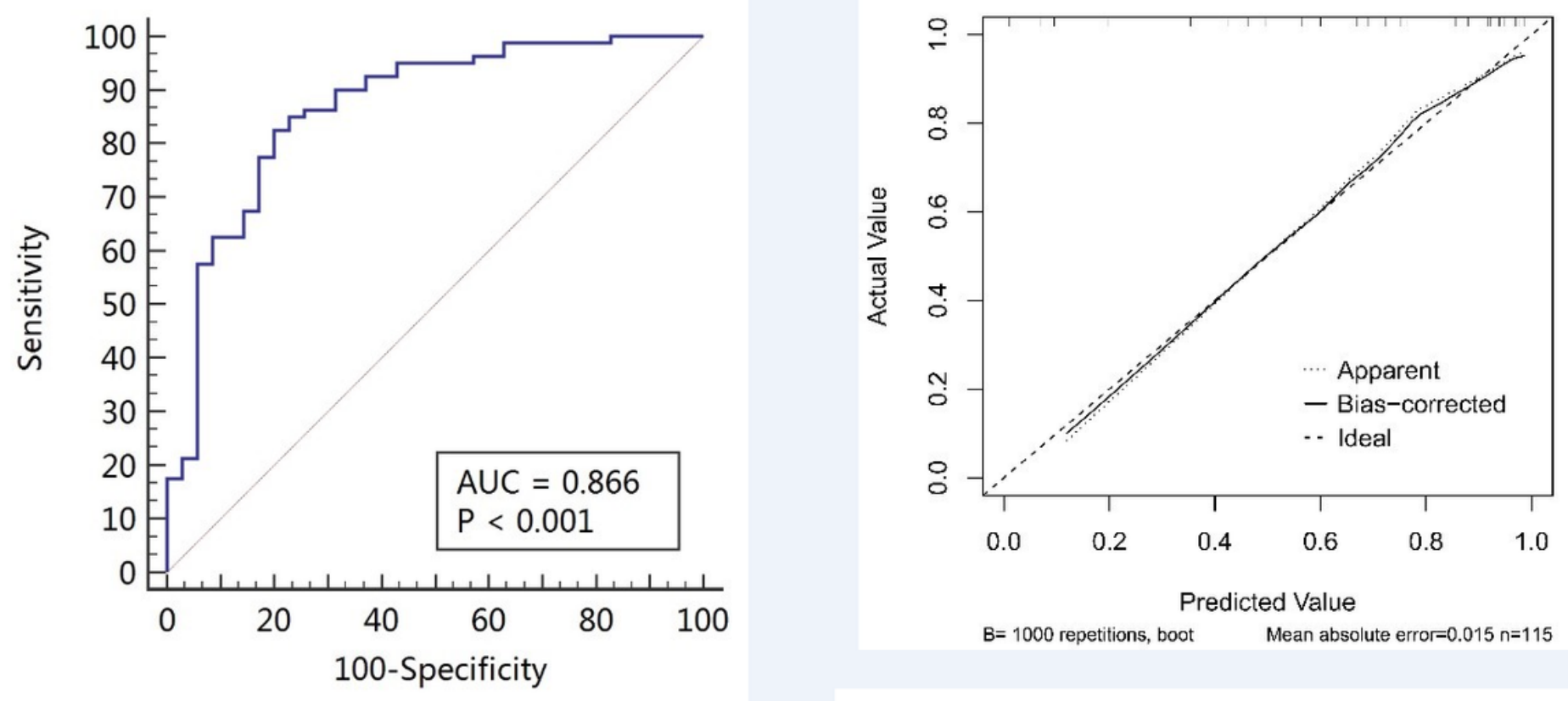

Figure 4

ROC analysis of the prediction model using $\mathrm{N}$ stage and RRS parameters. RRS, Radiomics Risk Score; $\mathrm{ROC}$, receiver operating characteristic 


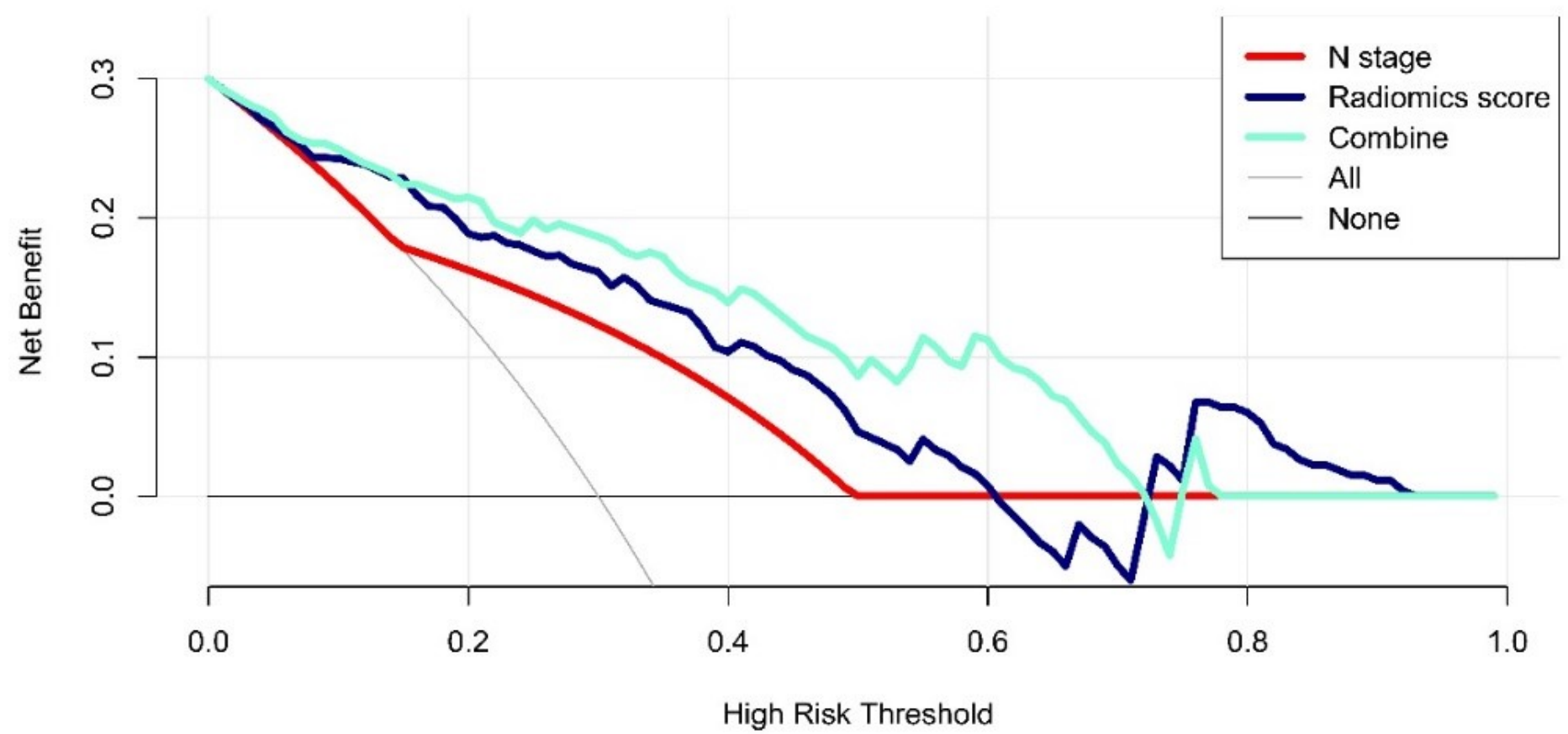

Figure 5

Decision curve analysis of the radiomics model The y axis measures the net benefit. The red and blue lines represent the $\mathrm{N}$ stage and RRS, respectively. The light green line represents the $\mathrm{N}$ stage and RRS combination.

\section{Supplementary Files}

This is a list of supplementary files associated with this preprint. Click to download.

- Table1.pdf

- Table2.pdf 